\title{
Prenatal diagnosis and genetic counseling in a fetus associated with risk of Angelman syndrome with a small supernumerary marker chromosome derived from chromosome 22
}

\author{
Yu-an $\mathrm{Hu}^{\dagger}$, Yingxia Cui ${ }^{\dagger}$, Xiaobo Fan, Qiuyue WU, Weiwei Li and Weiping Wang*
}

\begin{abstract}
Background: Angelman syndrome (AS) is a neurodevelopmental disorder. AS patients concomitant with SSMC are rather rare events. It will provide more useful and proper information for genetic counseling to identify the SSMC origin.

Case presentation: A 27-year-old woman was referred for genetic counseling and prenatal diagnosis at 26 weeks of gestation due to her elder daughter, diagnosed as Angelman syndrome (AS) with an interstitial deletion in one of the chromosomes 15, carrying a small supernumerary marker chromosome (sSMC). The G-banding results of the woman and her current fetus both were 47,XX,+mar. In this paper, fluorescence in situ hybridization (FISH) results showed that there was no deletion of chromosome 15 in the woman and fetus. We demonstrated that the proband's SSMC was maternally inherited and was an inv dup(22)(q11.1), and that the deletion in 15q11.2-q13.1 was de novo.

Conclusions: Taking into account above results and normal phenotypes of the proband's mother, in this case we suggest that the SSMC don't increase the recurrence risk of AS. After prenatal diagnosis, the woman chose to continue the pregnancy, and finally gave birth to a normal female infant.
\end{abstract}

Keywords: Angelman syndrome, Small supernumerary marker chromosomes (SSMC), Genetic counseling, Prenatal diagnosis

\section{Background}

Angelman syndrome (AS) is a neurodevelopmental disorder. Main clinical characteristics of AS include severe developmental delay, speech impairment, movement or balance disorder and apparent happy demeanor. Genetic mechanisms of AS involved the commonest, (micro) deletions in maternal chromosome 15q11-13, paternal uniparental disomy 15 (UPD), imprinting defects and/or mutation in the disease-causing gene $U B E 3 A$ (ubiquitin protein ligase E3A) [OMIM:105830] [1].

Small supernumerary marker chromosomes (sSMC) are defined as structurally abnormal chromosomes that cannot be identified or characterized unambiguously by

\footnotetext{
* Correspondence: wangwp_nj@163.com

${ }^{\dagger}$ Equal contributors

Institute of Laboratory Medicine, Jinling Hospital, Nanjing University School

of Medicine, 305 East Zhongshan Road, Nanjing, Jiangsu 210002, China
}

conventional banding cytogenetics alone. The size of sSMC is generally equal to or smaller than a chromosome 20 of the same metaphase spread [2]. The incidence has been estimated to be $0.075 \%$ in unselected prenatal cases and $0.044 \%$ in newborn infants, but evelated to $0.288 \%$ in mentally retarded patients [3]. Furthermore, $70 \%$ of sSMC are derived from acrocentric chromosomes [2].

As far as we know, there were rare nine AS cases reported in connection with sSMC. All AS cases with sSMC reported were related to chromosome 15 . It is generally considered that the sSMC derived from chromosome 15 and contained the Prader-Willi/Angelman syndrome critical region (PWACR) would cause clinical phenotypes. We reported a previous case of AS with sSMC derived not from chromosome 15 [4]. At that time, we excluded the possibility of paternally- inherited origin of sSMC, but couldn't deduce whether the origin of sSMC was de novo 
or maternally inherited. In this paper, the proband's mother gave her consent to genetic testing and counselled for her fetus. In order to evaluate the recurrence risk of AS correctly, we made clear that the proband's SSMC was maternally inherited and was an inv $\operatorname{dup}(22)(\mathrm{q} 11.1)$, and that the deletion in 15q11.2-q13.1 was de novo. Prenatal diagnosis and genetic counseling was performed in view of the findings.

\section{Case presentation}

A 27-year-old woman was referred for genetic counseling and prenatal diagnosis at 26 weeks of gestation because her first daughter was diagnosed as Angelman syndrome (AS) due to a $5.058 \mathrm{Mb}$ deletion in chromosome band 15q11.2-q13.1 and with a small supernumerary marker chromosome (sSMC). The proband was born by the woman and her former husband. Neither with her former husband nor with her current husband, they were in a non-consanguineous marriage. There was no family history of miscarriage or congenital malformations from either the two-term husbands or the woman. No abnormal symptoms were observed, and physical examination revealed that the couple was phenotypically normal.

The proband was diagnosed as AS at 3-year-old. Gbanding revealed a karyotype $47, \mathrm{XX},+$ mar in all of the peripheral blood lymphocytes. Her paternal karyotype was normal and the information of her mother was unknown formerly. Silver staining for the nucleolus organizer regions (NOR staining) revealed two satellites in both ends of the sSMC. Fluorescence in situ hybridization (FISH) using the 15 dual color DNA probes (Vysis, USA), which hybridize to D15S10,UBE3A and centromere of chromosome 15 confirmed the deletion of chromosome 15q11-13 and the sSMC was unrelated with chromosome 15.

\section{Material and methods \\ Cytogenetic analysis}

Peripheral blood from the woman and cord blood from the fetus were drawn for cytogenetic analysis including G-banding and fluorescence in situ hybridization (FISH). FISH using the 15 dual color DNA probes (Vysis, USA) was performed according to standard procure. Centromeric FISH probes for chromosome 14 and 22, followed by sub-centromeric FISH probes for chromosome 14 or 22 were adopted to identify the the origin of sSMC in the proband. Centromere FISH and sub-centromere FISH were kindly performed by the laboratory of Professor Thomas Liehr (Jena University Hospital, Friedrich Schiller University, Institute of Human Genetics).

\section{Results}

G-banding showed both of karyotypes in woman and her fetus were $47, \mathrm{XX},+$ mar in $100 \%$ of the analyzed cells.. This revealed the small supernumerary marker chromosome (sSMC) in the proband was maternally inherited. Fluorescence in situ hybridization confirmed that there was no deletion of chromosome 15q11-13 in the woman and her fetus, therefore Angelman syndrome
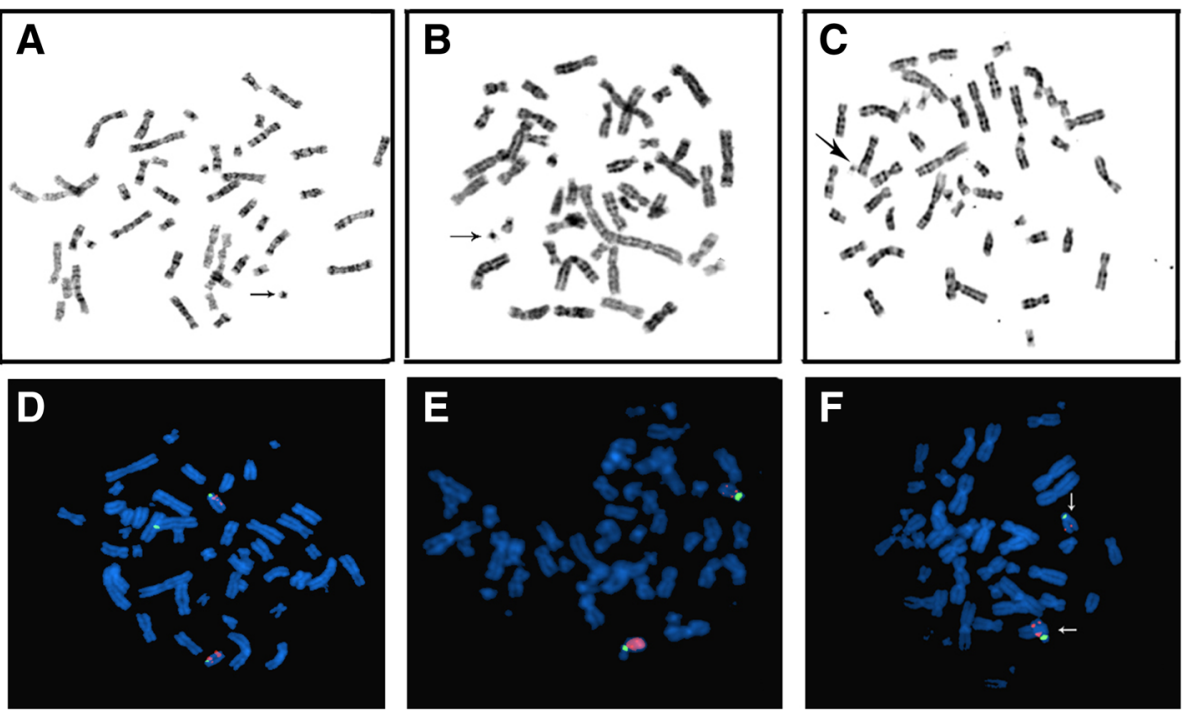

Fig. 1 Results of karyotypes and FISH using the 15 dual color DNA probes. a-c: The results of karyotypes from the mother (a), the fetus (b) and the proband (c) showed the SSMC in the proband and the fetus were maternally inherited. The black arrow indicates the SSMC. $\mathbf{d}-\mathbf{f}$ : The results of FISH using the 15 dual color DNA probes showed that there was no deletion in chromosome 15q11-13 observed both in the mother (d) and in the fetus (e) and that there was no additional centromeric signal of chromosome 15 in the three cases. The white $\operatorname{arrow}(\downarrow)$ shows the loss of chromosome 15q11-13 and the white arrow to left $(\leftarrow)$ indicates normal chromosome 15 in the proband (f). The orange signals indicate chromosome 15q11-13 and 15q22-24(used as control probe), the green signal indicates the centromere of chromosome 15. a, d: the mother of proband; $\mathbf{b}$, e: the fetus; $\mathbf{c}$, $\mathbf{f}$ : the proband 
was not considered. FISH analysis with centromeric probe of chromosome 15 also excluded the possibility of the origin from the chromosome 15 (Fig. 1).

Centromeric FISH and sub-centromeric FISH on the proband's metaphases spreads demonstrated that the sSMC originated from chromosome 22 and was a variant of inv dup(22)(q11.1) (Fig. 2), thus the final karyotype of the proband was written as $47, \mathrm{XX}, \operatorname{del}(15)(\mathrm{q} 11 \mathrm{q} 13)$, +inv $\operatorname{dup}(22)(\mathrm{q} 11.1)$.

\section{Discussion}

Angelman syndrome (AS) has a prevalence of 1/10,000 $1 / 20,000$ individuals [1], it could be clinically diagnosed by a serial of characteristic features including developmental delay, intellectual disability, speech impairment, seizures, movement or balance disorder, apparent happy demeanor, and so on. Genetic analysis, such as karyotype, fluorescence in situ hybridization (FISH), methylation analysis of the chromosome 15q11-13 region and chromosomal microarrays analysis might be helpful for the diagnosis of AS and provide information as much as possible for genetic counseling. In our previous report, Affymetrix cytogenetics whole genome $2.7 \mathrm{M}$ arrays were applied to demonstrate a 5.058 Mb deletion in chromosome band 15q11.2q13.1 between positions chr15: 21,170,573-26,229,285 bp, in which 10 genes (SNORD116-14, SNORD115-30, UBE3A, SNORD109B, SNORD115-23, SNORD116-9, SNORD116-16, SNORD115-10, GABRG3, SNORD116-2) were contained. The microarrays analysis further characterized the region confirmed by FISH.

What's special about the AS proband we reported was accompanied by a small supernumerary marker chromosome (sSMC). AS patients concomitant with SSMC are rather rare events. Just nine AS cases with the SSMC were reported by now. As summarized in sSMC-homepage (http://ssmc-tl.com/sSMC.html), the corresponding sSMC were derived from chromosome 15 in all nine cases. In five of them, the AS was due to a paternal UPD 15, in three due to a deletion in the AS-critical region [5-8] and in the remainder case the molecular result for AS was not elucidated. Chromosome 15 is the most common origin of sSMC in human [2,3]. Summarizing data indicated that the majority of sSMCs $(65 \%)$ originated from chromosome 15, while sSMCs derived from other acrocentric chromosomes 13, 14, 21, and 22 constitute only $7 \%$ [9]. When AS accompanied by sSMC, particularly contained the Prader-Willi/Angelman syndrome critical region in chromosome 15, the correlation between the genotype and the clinical phenotype became more complex [10]. The abnormal phenotype was associated with the origin and genetic nature of sSMC. It is important to identify the chromosome origin if the sSMC is visible in the karyotype of AS.

In our case, karyotypes showed the sSMC was maternally inherited. Furthermore, FISH with centromeric probe of chromosome 15 revealed that the SSMC was unrelated to chromosome 15. Finally, Centromeric FISH and sub-centromeric FISH were carried out to confirm that the sSMC derived from chromosome 22 and was an inv $\operatorname{dup}(22)(q 11.1)$ (Fig. 2). Additionally, data from Affymetrix cytogenetics whole genome $2.7 \mathrm{M}$ arrays did not indicate the origin information. We speculated that the genetic material nature of the $\mathrm{sSMC}$ was heterochromatin and there was no annotated gene present corresponding to the arrays. Thus, the proband we reported previously, to our knowledge, is the first AS case with the maternally inherited sSMC derived from chromosome 22.

The risk to the sibs of an individual with AS who was identified a de novo large deletion in the chromosome $15 \mathrm{q} 11-13$ is reported $<1 \%$ [11]. However, it is estimated that there were about $2.7 \times 10^{6}$ living sSMC carriers in the world, almost $70 \%$ of those are clinically normal [12]. Furthermore, in $70 \%$ of the carriers the sSMC is de novo, in $20 \%$ inherited from the mother, and in $10 \%$ inherited from the father. Familial sSMCs in general have little or no effect on the phenotype if the parent's development is normal [13].

Taking into account the normal phenotype of the proband's mother, we thought that AS in the proband could be explained by the microdeletion on one of the chromosome 15q11-13, and not by the presence of the sSMC.

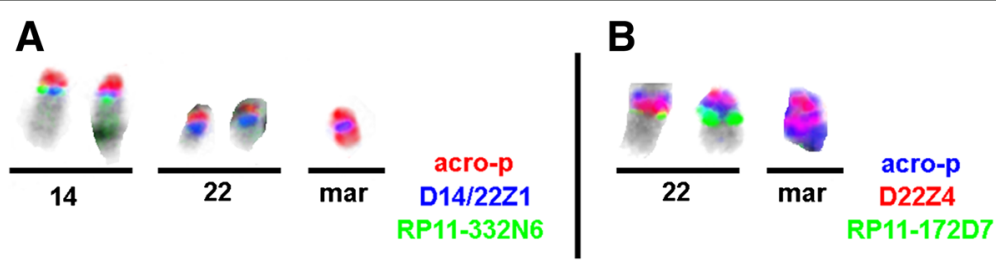

Fig. 2 The origin of marker chromosome characterized by centromeric FISH and subcentromeric FISH. a: The sSMC wasn't derived from chromosome 14. The centromeric probe specific for chromosome 14 / 22(D14/22Z1 blue) and a probe specific for all acrocentric p-arms (acro-p red) were present on the marker chromosome. But the centromere-near probe in 14q11.2(RP11-332 N6 green) was not detected on the marker chromosome. b: The sSMC originated from chromosome 22 and was an inv dup(22)(q11.1). The centromeric probe specific for chromosome 22(D22Z4 red) and a probe specific for all acrocentric p-arms (acro-p blue) were present on the marker chromosome. A centromere-near probe in 22q11.21(RP11-172D7 green) was not detected.14:chromosome 14; 22: chromosome 22; mar: SSMC 
After prenatal diagnosis and genetic counseling, the woman chose to continue the pregnancy, and finally gave birth to a normal female infant.

\section{Conclusion}

In summary, the recurrence risk of AS depends on the genetic mechanism of AS in the proband. When AS accompanied by sSMC, it will provide more useful and proper information for genetic counseling to identify the sSMC origin.

\section{Consent}

Written informed consent was obtained from the patient for publication of this case report and any accompanying images. A copy of the written consent is available for review by the Editor-in-Chief of this journal.

\section{Competing interests}

The authors declare that they have no competing interests.

\section{Authors' contributions}

$Y A H$ and XBF played a role in the molecular genetic experiments and drafted the manuscript. YXC had major roles in the design of the study and analysis of clinical data. QYW and WWL participated in karyotypes analysis. WPW participated in the design and coordination of the study. All authors read and approved the final manuscript.

\section{Acknowledgments}

We heartily appreciate the help from Professor Thomas Liehr. We would like to thank the proband and her family for their collaboration.

This study is supported by Nanjing Science and Technology Development Project (No.201402008), Medical and Health Research Foundation of Nanjing Military Region (No.15MS125), foundation of Jinling Hospital of Nanjing University School of Medicine $(2014044,2015046)$

Received: 10 November 2015 Accepted: 22 April 2016

Published online: 03 May 2016

\section{References}

1. Bird LM. Angelman syndrome: review of clinical and molecular aspects[J]. Appl Clin Genet. 2014;7:93-104.

2. Liehr T, Ewers E, Kosyakova N, et al. Handling small supernumerary marker chromosomes in prenatal diagnostics[J]. Expert Rev Mol Diagn. 2009;9(4):317-24.

3. Liehr $\mathrm{T}$, Weise A. Frequency of small supernumerary marker chromosomes in prenatal, newborn, developmentally retarded and infertility diagnostics[J]. Int J Mol Med. 2007;19(5):719-31.

4. Fan XB, Cui YX, Zhou YC, et al. Cytogenetic and molecular genetic diagnosis for a girl with Angelman syndrome and patent ductus arteriosus. Chin J Clin Lab Sci. 2011;1:66-8.

5. Roberts S, Maggouta F, Thompson R, et al. A patient with a supernumerary marker chromosome (15), Angelman syndrome, and uniparental disomy resulting from paternal meiosis II non-disjunction[J]. J Med Genet. 2002; 39(2):E9.

6. Thompson RJ, Bolton PF. Case report: Angelman syndrome in an individual with a small SMC(15) and paternal uniparental disomy: a case report with reference to the assessment of cognitive functioning and autistic symptomatology[J]. J Autism Dev Disord. 2003;33(2):171-6.

7. Baumer A, Wiedemann U, Hergersberg M, et al. A novel MSP/DHPLC method for the investigation of the methylation status of imprinted genes enables the molecular detection of low cell mosaicisms[J]. Hum Mutat. 2001;17(5):423-30.

8. Liehr T, Klein E, Mrasek K, et al. Clinical impact of somatic mosaicism in cases with small supernumerary marker chromosomes[J]. Cytogenet Genome Res. 2013;139(3):158-63.
9. Liehr T. Small Supernumerary Marker Chromosomes (sSMC): A Guide for Human Geneticists and Clinicians.[Z]. Heidelberg, Dordrecht, London, New York: Springer; 2011

10. Koç A, Onur SÖ, Ergün MA, et al. Supernumerary marker chromosome 15 in a male with azoospermia and open bite deformity[J]. Asian J Androl. 2009; 11(5):617-22.

11. Williams CA, Driscoll DJ, Dagli Al. Clinical and genetic aspects of Angelman syndrome[J]. Genet Med. 2010;12(7):385-95.

12. Liehr T, Mrasek K, Weise A, et al. Small supernumerary marker chromosomesprogress towards a genotype-phenotype correlation[J]. Cytogenet Genome Res. 2006:112(1-2):23-34.

13. Hashemzadeh-Chaleshtori M, Teimori H, Ghasemi-Dehkordi P, et al. Small supernumerary marker chromosomes and their correlation with specific syndromes[J]. Adv Biomed Res. 2015;4(1):140.
Submit your next manuscript to BioMed Central and we will help you at every step:

- We accept pre-submission inquiries

- Our selector tool helps you to find the most relevant journal

- We provide round the clock customer support

- Convenient online submission

- Thorough peer review

- Inclusion in PubMed and all major indexing services

- Maximum visibility for your research

Submit your manuscript at www.biomedcentral.com/submit 